\title{
Empirical prescribing of penicillin G/N reduces risk of readmission of hospitalized patients with community-acquired pneumonia in Norway: a retrospective observational study
}

\author{
June Utnes Høgli ${ }^{1,2}$, Beate Hennie Garcia ${ }^{2}$, Kristian Svendsen ${ }^{2,3}$, Vegard Skogen ${ }^{4,5,6}$ and Lars Småbrekke $2^{2^{*}}$ (1)
}

\begin{abstract}
Background: Norwegian guideline recommendations on first-line empirical antibiotic prescribing in hospitalised patients with community-acquired pneumonia (CAP) are penicillin GN in monotherapy, or penicillin G in combination with gentamicin (or cefotaxime) in severely ill patients. The aim of this study was to explore how different empirical antibiotic treatments impact on length of hospital stay (LOS) and 30-day hospital readmission. A secondary aim was to describe median intravenous- and total treatment duration.

Methods: We included CAP patients ( $\geq 18$ years age) hospitalised in North Norway during 2010 and 2012 in a retrospective study. Patients with negative chest $x$-ray, malignancies or immunosuppression or frequent readmissions were excluded. We collected data on patient characteristics, empirical antibiotic prescribing, treatment duration and clinical outcomes from electronic patient records and the hospital administrative system. We used directed acyclic graphs for statistical model selection, and analysed data with mulitvariable logistic and linear regression.

Results: We included 651 patients. Median age was 77 years [IQR; $64-84]$ and 46.5\% were female. Median LOS was 4 days [IQR; 3-6], 30-day readmission rate was $14.4 \%$ and 30-day mortality rate was $6.9 \%$. Penicillin GN were empirically prescribed in monotherapy in $51.5 \%$ of patients, penicillin $\mathrm{G}$ and gentamicin in combination in $22.9 \%$ and other antibiotics in $25.6 \%$ of patients. Prescribing other antibiotics than penicillin GN monotherapy was associated with increased risk of readmission [OR 1.9, 95\% Cl; 1.08-3.42]. Empirical antibiotic prescribing was not associated with LOS. Median intravenous- and total treatment duration was 3.0 [IQR; 2-5] and 11.0 [IQR; 9.8-13] days.

\footnotetext{
* Correspondence: lars.smabrekke@uit.no

${ }^{2}$ Department of Pharmacy, Faculty of Health Sciences, UiT - The Arctic University of Norway, N-9037 Tromsø, Norway

Full list of author information is available at the end of the article
}

C C The Author(s). 2020 Open Access This article is licensed under a Creative Commons Attribution 4.0 International License, which permits use, sharing, adaptation, distribution and reproduction in any medium or format, as long as you give appropriate credit to the original author(s) and the source, provide a link to the Creative Commons licence, and indicate if changes were made. The images or other third party material in this article are included in the article's Creative Commons licence, unless indicated otherwise in a credit line to the material. If material is not included in the article's Creative Commons licence and your intended use is not permitted by statutory regulation or exceeds the permitted use, you will need to obtain permission directly from the copyright holder. To view a copy of this licence, visit http://creativecommons.org/licenses/by/4.0/ The Creative Commons Public Domain Dedication waiver (http://creativecommons.org/publicdomain/zero/1.0/) applies to the data made available in this article, unless otherwise stated in a credit line to the data. 
(Continued from previous page)

Conclusions: Our findings show that empirical prescribing with penicillin GN in monotherapy in hospitalised nonsevere CAP-patients, without complicating factors such as malignancy, immunosuppression and frequent readmission, is associated with lower risk of 30-day readmission compared to other antibiotic treatments. Median total treatment duration exceeds treatment recommendations.

Keywords: Community-acquired pneumonia, Antibiotics, Guideline, Clinical outcome, Norway, Antibiotic stewardship program

\section{Background}

Community-Acquired Pneumonia (CAP) is the leading cause of death due to infectious diseases in adults worldwide. The annual adult incidence range from 1 to 8 per 1000 inhabitants, is higher in men and increases with age. The 30-day hospital readmission rate range from 15 to $20 \%$ [1-5]. Reported 30-day mortality rate due to CAP in Scandinavia ranges from 7 to $11 \%[3,6]$.

Streptococcus pneumoniae is the most frequent identified cause of CAP. Other common pathogens include Haemophilus influenzae, Mycoplasma pneumoniae and respiratory viruses $[3,7-9]$. Obtaining a microbiological diagnosis is difficult, and an aetiological diagnosis in CAP is unconfirmed in up to $50 \%$ of patients [7-9]. In Norway, $<1 \%$ of S.pneumoniae blood culture and respiratory isolates are resistant for penicillin G/V, and 6 and $8.2 \%$ of S.pneumoniae in blood culture- and respiratory isolates are resistant to erythromycin, respectively [10]. For H.influenzae blood culture isolates the prevalence of beta-lactamase and chromosomal resistance are 17.8 and $16.1 \%$, respectively [10].

Appropriate treatment for CAP is reflected by recommendations in clinical practice guidelines (CPGs). Geographic location and host factors predict the causative pathogen and antimicrobial resistance (AMR). Consequently, recommendations in CPGs can differ between countries. In most European and American guidelines a $\beta$-lactam (type of recommended $\beta$-lactam differs between countries) combined with a macrolide, or a respiratory fluoroquinolone in monotherapy, is recommended as empirical treatment for hospitalised CAP-patients [11-13]. Scandinavian and Dutch guidelines recommends narrow spectrum penicillin G/V (or ampicillin) in monotherapy as first-line empirical treatment in non-severe CAP with no routinely empirical coverage for atypical pathogens [14-17]. Recommendations for severely ill CAP patients varies, and the Norwegian guideline recommends penicillin $G$ in combination with gentamicin or cefotaxime in monotherapy for patients where atypical pathogens are not suspected [15].

Appropriate antibiotic prescribing is essential for patient safety and outcome, and for reducing emergence of AMR [18]. A Danish study recently found no association between empirical treatment with penicillin $G / V$ and mortality in mild to moderate CAP [3]. Inappropriate prolonged treatment has been associated with longer LOS, higher costs and an increase in adverse drug reactions without altering treatment effect, number of recurrent infections and mortality $[19,20]$.

The aim of this study was to explore how different empirical antibiotic treatments impact on LOS and 30-day hospital readmission. In addition, we aimed to describe median intravenous (IV) and total treatment duration.

\section{Methods}

\section{Setting and study population}

The University hospital of North Norway (UNN) is a 500-bed hospital in the North Norway health region. UNN serves about 195,000 inhabitants and is divided into three subunits located in three different towns; Harstad, Narvik and Tromsø.

We conducted a retrospective observational study including patients $\geq 18$ years discharged from UNN during 2010 and 2012 with a CAP diagnosis registered at discharge (ICD-10 codes J13-16 or J18). We excluded patients with no confirmed chest $\mathrm{x}$-ray, nosocomial or aspiration pneumonia, immunosuppression or malignancies, (suspected) co-infection, discharged from surgical departments, transferred from or to other hospitals and with consecutive admissions due to CAP the current year (patients could only be included once per year in the study), see Fig. 1.

\section{Data collection and clinical definitions}

We retrospectively extracted the following patient data from electronic records, medication charts and laboratory data; age, gender, antibiotics used pre-hospitalisation (yes/ no), nursing home residency status, penicillin allergy status, relevant comorbidities (chronic obstructive pulmonary disease (COPD), heart failure and diabetes mellitus I or II), infection-relevant clinical and laboratory data from the first 3 days of hospitalisation (blood pressure, heart rate, respiratory rate, body temperature, oxygen saturation, leucocytes and c-reactive protein), microbiological tests ordered and pathogens identified. We calculated severity according to CRB-65 (confusion, respiratory rate, blood pressure, age $\geq 65$ years) based on information at admission [21]. 


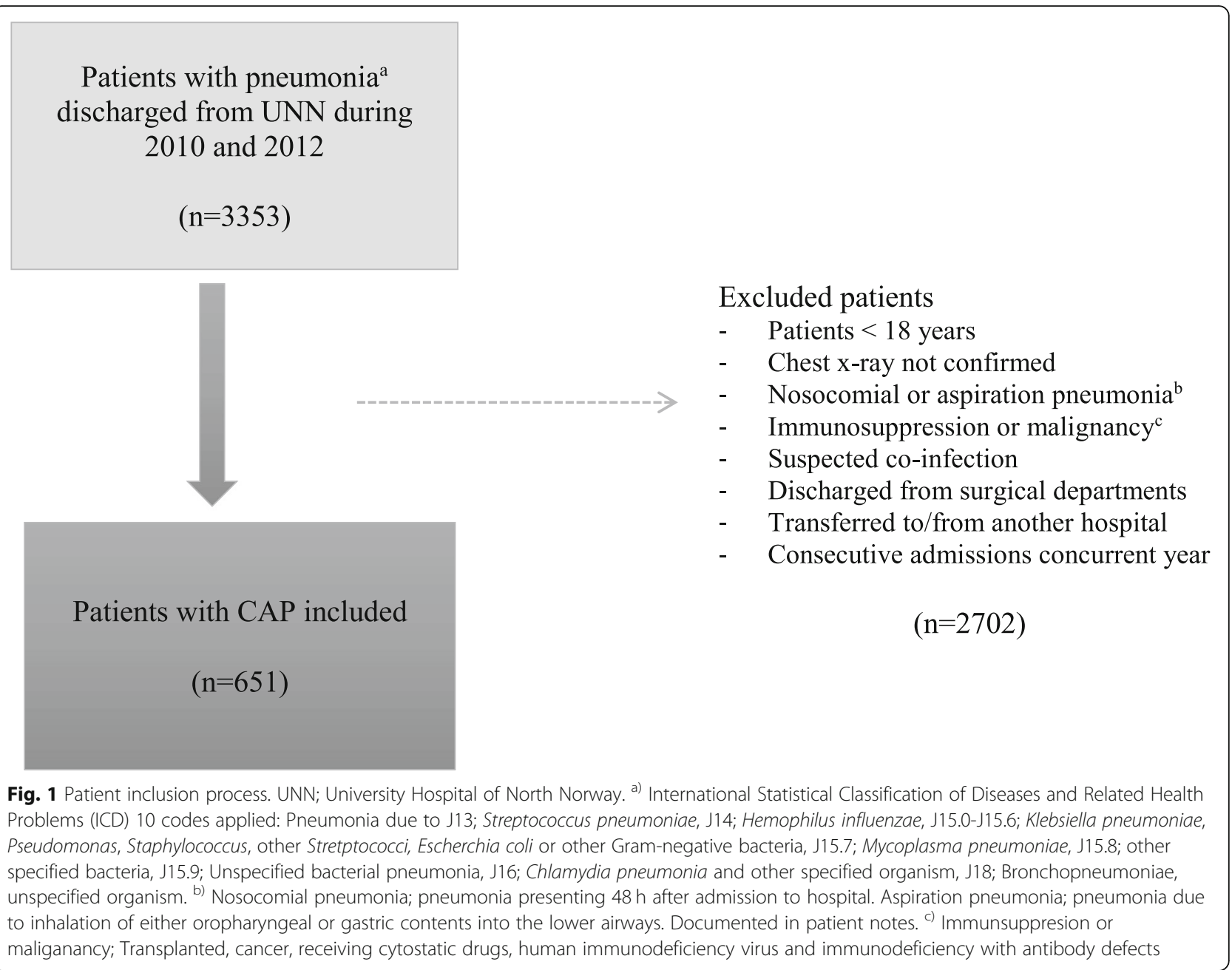

We collected the complete antibiotic medication list for the hospital stay (drug, dose, route of administration and duration), including any amendment of empirical antibiotic prescribing within first 3 days (i.e. switch to a broader spectrum IV antibiotic, or addition of a new antibiotic to the current regime). We defined total treatment duration as length of antibiotic treatment in hospital plus the prescribed length of treatment at discharge. Antibiotic treatment started pre-hospitalisation was not included in calculation of treatment duration. The clinical outcome measures LOS, allcause 30-day readmission (unplanned, calculated from time of discharge) and all-cause 30-day mortality were collected from the hospital administrative system.

Patients were categorized into three groups according to type of empirical antibiotic prescribed:1) penicillin $\mathrm{G} / \mathrm{V}$ in monotherapy, 2) penicillin $G$ in combination with gentamicin and 3) all other antibiotic treatments.

\section{Statistics}

We used Microsoft ${ }^{\circ}$ Office Excel 2010 and STATA ${ }^{\circ} 14$ for data analysis. Descriptive statistics are reported as counts and percentages for categorical data, and median and 25th to 75th interquartile range for continuous data. We performed descriptive statistics on the crude study population and a stratified analysis on patient characteristics and clinical outcomes for the three different empirical antibiotic treatment categories. In the stratified analysis/group comparison, we tested difference in proportions (categorical data) with Pearson's $\mathrm{X}^{2}$-test and difference in means (continuous data) with ANOVA test. A $P$-value of $<0.05$ was considered statistically significant.

We used multivariable linear regression to explore the impact of empirical antibiotic prescribing (exposure) on LOS (outcome), and logistic regression to explore the impact of empirical antibiotic prescribing (exposure) on 30-day readmission (outcome).

Directed Acyclic Graphs (DAGs) were applied for statistical model selection [22]. The DAGs were created and analysed in the browser-based program DAGitty version 2.0. (http://www.dagitty.net), see Additional file 1. We adjusted for the following confounders; CRB-65, 
Table 1 Patient characteristics $(n=651)$

\begin{tabular}{|c|c|c|}
\hline \multirow[t]{2}{*}{ Patient characteristics } & \multicolumn{2}{|c|}{ Total } \\
\hline & $\mathrm{n}$ & $(\%)$ \\
\hline Gender, female & 303 & $(46.5)$ \\
\hline Age, years, median (IQR) & 77 & $(64-84)$ \\
\hline Nursing home residents & 70 & $(10.8)$ \\
\hline Penicillin allergy ${ }^{a}$ & 70 & $(10.8)$ \\
\hline \multicolumn{3}{|l|}{ Comorbidities } \\
\hline COPD & 205 & $(31.5)$ \\
\hline Heart failure & 140 & $(21.5)$ \\
\hline Diabetes mellitus I or II & 85 & $(13.1)$ \\
\hline \multicolumn{3}{|l|}{ CRB-65 score ${ }^{b}$} \\
\hline 0 & 110 & $(16.9)$ \\
\hline 1 & 225 & $(34.9)$ \\
\hline 2 & 179 & $(27.5)$ \\
\hline 3 & 43 & $(6.6)$ \\
\hline 4 & 6 & $(0.9)$ \\
\hline Missing data & 88 & $(13.5)$ \\
\hline Registered in admission notes & 1 & $(0.2)$ \\
\hline Antibiotic use pre-hospitalization & 171 & $(26.3)$ \\
\hline \multicolumn{3}{|l|}{ Empirical antibiotic prescribing } \\
\hline Penicillin GN monotherapy & 335 & $(51.5)$ \\
\hline Penicillin G + gentamicin & 149 & $(22.9)$ \\
\hline Other antibiotics & 167 & $(25.7)$ \\
\hline \multicolumn{3}{|l|}{ Treatment duration } \\
\hline IV treatment duration, median (IQR) & 3 & $(2-5)$ \\
\hline Total treatment duration, median (IQR) & 11 & $(9.8-13)$ \\
\hline \multicolumn{3}{|l|}{ Microbiological diagnostics } \\
\hline Blood culture & 499 & $(76.7)$ \\
\hline Nasopharynx & 179 & $(27.5)$ \\
\hline Expectorate & 100 & $(15.4)$ \\
\hline Pneumococcal urinary antigen test & 327 & $(50.2)$ \\
\hline Legionella urinary antigen test & 51 & $(7.8)$ \\
\hline Serology M. or C.pnuemoniae & 19 & $(2.9)$ \\
\hline Other & 220 & $(33.8)$ \\
\hline None & 68 & $(10.4)$ \\
\hline \multicolumn{3}{|l|}{ Aetiology } \\
\hline S.pneumoniae & 61 & $(9.4)$ \\
\hline M. or C. pneumoniae & 22 & $(3.4)$ \\
\hline H.influenzae & 11 & $(1.7)$ \\
\hline S.aureus & 7 & $(1.1)$ \\
\hline Other bacteria & 14 & $(2.2)$ \\
\hline Influenza virus A or B & 12 & $(1.8)$ \\
\hline Other respiratory viruses & 18 & $(2.8)$ \\
\hline None identified & 514 & (79.0) \\
\hline
\end{tabular}

Clinical outcomes
Table 1 Patient characteristics $(n=651)$ (Continued)

\begin{tabular}{|c|c|c|}
\hline \multirow[t]{2}{*}{ Patient characteristics } & \multicolumn{2}{|c|}{ Total } \\
\hline & $\bar{n}$ & $(\%)$ \\
\hline 30-day readmission & 90 & $(14.4)$ \\
\hline Length of stay in hospital, median & 4 & $(3-6)$ \\
\hline 30-day mortality & 44 & $(6.9)$ \\
\hline \multicolumn{3}{|c|}{$\begin{array}{l}C R B-65 \text { Confusion, respiration, blood pressure and age } \geq 65 y, C O P D \text { Chronic } \\
\text { obstructive pulmonary disease, } I Q R \text { inter quartile range, } I V \text { intravenous } \\
\text { a Documented penicillin allergy in patient notes to beta-lactams } \\
\text { b }{ }^{\text {S }} \text { coring made retrospectively based on admission data/journal data }\end{array}$} \\
\hline
\end{tabular}

comorbidities, age, gender, pathogen, year of admittance, hospital and nursing home residence. LOS, 30-day readmission, IV- and total treatment duration were recorded as missing for patients who died in hospital. Only patients with complete data on outcome and confounders were included in the regression analyses.

\section{Results}

Study population and outcomes

We included 651 (19.4\%) out of the 3342 patients that were discharged with ICD-10 code J13-16 or J18. Median age was 77 years (IQR; 64-84] and $46.5 \%$ were female. An aetiological agent was identified in $21 \%$ of patients, and S.pneumoniae was the most common pathogen detected. About $11 \%$ of patients were allergic to penicillin. Median LOS was 4 days [IQR; 3-6], and the 30 -day readmission rate was $14.4 \%$. The 30 -day mortality rate was $6.9 \%$. We calculated that $7.5 \%$ of patients had high risk of mortality according to CRB-65 (score $\geq$ 3). See Table 1.

\section{Antibiotic prescribing}

Penicillin G/V were empirically prescribed in monotherapy in $51.5 \%$ of patients, penicillin G and gentamicin in combination in $22.9 \%$ and other antibiotics in $25.6 \%$ of patients. Cefotaxime, doxycycline and erythromycin were the most commonly prescribed antibiotics among those receiving other antibiotics. See Additional file 2 for a description of all empirical antibiotics prescribed and Additional file 3 for a description of choice of empirical antibiotic versus subsequent bacterial pathogen identified. Empirical antibiotic prescribing was amended before day three in $16.3 \%$ of the patients. Median IV treatment duration was 3.0 days (mean 3.7) and median total treatment duration was 11.0 days (mean 11.6).

Group comparison of three different empirical treatments Stratified results on patient characteristics and clinical outcomes for the different empirical antibiotic treatments are given in Table 2. The results show that patients prescribed 'other antibiotic treatments' had higher 30-days readmittance rate, had higher prevalence of 
Table 2 Stratified results for different empirical prescribing. Unadjusted analysis

\begin{tabular}{|c|c|c|c|c|}
\hline & $\begin{array}{l}\text { Monotherapy penicillin } \\
\mathrm{G} N(n=335)\end{array}$ & $\begin{array}{l}\text { Combination penicillin G } \\
\text { and gentamicin }(n=149)\end{array}$ & $\begin{array}{l}\text { Other antibiotics } \\
(n=167)\end{array}$ & $p$-value* \\
\hline & n (\%) & n (\%) & n (\%) & \\
\hline \multicolumn{5}{|l|}{ Age, years } \\
\hline Mean & 72.8 & 70.8 & 70.4 & 0.29 \\
\hline Gender, female & $150(44.8)$ & $67(45.0)$ & $86(51.5)$ & 0.33 \\
\hline \multicolumn{5}{|l|}{ Comorbidities } \\
\hline COPD & $94(28.1)$ & $44(29.5)$ & $67(40.1)$ & 0.02 \\
\hline Heart failure & $73(21.8)$ & $26(17.5)$ & $41(24.6)$ & 0.30 \\
\hline Diabetes mellitus I or II & $50(14.9)$ & $13(8.7)$ & $22(13.2)$ & 0.17 \\
\hline Nursing home resident & $27(8.1)$ & $22(14.8)$ & $21(12.6)$ & 0.06 \\
\hline Penicillin allergy & $8(2.4)$ & $2(1.3)$ & $60(35.9)$ & 0.00 \\
\hline CRB-65 & & & & 0.11 \\
\hline 0 & $57(17.0)$ & $23(15.4)$ & $30(18.0)$ & \\
\hline 1 & $127(37.9)$ & $38(25.5)$ & $60(35.9)$ & \\
\hline 2 & $87(26.0)$ & $46(30.9)$ & $46(27.5)$ & \\
\hline 3 & $15(4.5)$ & $17(11.4)$ & $11(6.6)$ & \\
\hline 4 & $2(0.6)$ & $2(1.3)$ & $2(1.2)$ & \\
\hline Missing data & $47(14.0)$ & $23(15.4)$ & $18(10.8)$ & \\
\hline Antibiotic use pre-hospitalization & $64(19.1)$ & $40(26.9)$ & $67(40.1)$ & 0.00 \\
\hline Treatment amended within 3 days & $56(16.7)$ & $25(16.8)$ & $25(15.0)$ & 0.87 \\
\hline IV treatment duration, mean (days) & 3.4 & 4.1 & 4.1 & 0.01 \\
\hline Total treatment duration, mean (days) & 11.4 & 12.3 & 11.3 & 0.04 \\
\hline \multicolumn{5}{|l|}{ Clinical outcomes } \\
\hline 30-day readmission & $38(11.6)$ & $19(13.4)$ & $33(20.8)$ & 0.02 \\
\hline Length of stay (mean) & 5.1 & 5.8 & 5.0 & 0.20 \\
\hline 30-day mortality & $20(6.0)$ & $11(7.4)$ & $13(7.8)$ & 0.70 \\
\hline
\end{tabular}

CRB-65 Confusion, respiration, blood pressure and age $\geq 65 y$, COPD Chronic obstructive pulmonary disease, $I V$ intravenous

*Categorical data analyzed using Pearson's X2-test and continuous data using ANOVA test

COPD and penicillin allergy, and were more frequently prescribed antibiotics pre-hospitalisation. In addition, we observed differences in nursing home residency status and IV- and total treatment duration between the treatments. Patients prescribed other antibiotics did not have more severe CAP, measured by CRB-65, than patients prescribed penicillin $\mathrm{G} / \mathrm{V}$.

\section{Association with length of stay in hospital $(n=626)$}

Neither empirical prescribing of penicillin G/V in monotherapy, penicillin $\mathrm{G}$ in combination with gentamicin nor other antibiotic treatments were associated with LOS. A 1 year increase in age increased mean LOS with an average of 0.04 days [ $95 \% \mathrm{CI} ; 0.02,0.07]$. Heart failure was associated with an increase of 1.4 days in mean LOS [95\% CI; 0.6, 2.2], and a CRB-65 score of 3 was associated with an increase of 1.9 days in mean LOS [95\% CI; 0.4, 3.50]. Positive findings of S.pneumoniae, S.aureus and other bacteria was associated with 1.22 [95\% CI; $0.10,2.34], 4.10$ [95\% CI; 1.24, 6.96] and 5.19 [95\% CI; $2.98,7.40]$ days longer mean LOS, respectively. Admission to hospital in 2012 compared to 2010 was associated with $-0.42[95 \% \mathrm{CI}$; $-0.73,-0.11]$ shorter mean LOS. We also observed differences between hospitals. See Table 3 for the complete table with adjusted coefficients.

\section{Association with 30-day readmission $(n=609)$}

OR for 30-day readmission for prescribing 'other antibiotics' compared with penicillin G/V in monotherapy (reference) was 1.9 [95\% CI; 1.08-3.42]. OR for prescribing penicillin $\mathrm{G}$ in combination with gentamicin compared with penicillin G/V in monotherapy (reference) was 1.4 [95\% CI; 0.73-2.66].

Female patients had lower risk of 30-days hospital readmission [OR 0.5, 95\% CI; 0.30-0.82], while patients 
Table 3 Multivariable regression analysis. Association between empirical antibiotic prescribing, various covariates and length of hospital stay $(\operatorname{LOS})(n=626)$ and 30-day readmission $(n=609)$, respectively

\begin{tabular}{|c|c|c|c|c|}
\hline \multirow[t]{2}{*}{ Variable } & \multicolumn{2}{|c|}{ Length of hospital stay $^{a}$} & \multicolumn{2}{|l|}{ 30-day readmission ${ }^{b}$} \\
\hline & Adjusted coefficient & {$[95 \% \mathrm{Cl}]$} & Adjusted odds ratio & {$[95 \% \mathrm{Cl}]$} \\
\hline \multicolumn{5}{|l|}{ Empirical antibiotic prescribing } \\
\hline Penicillin GN in monotherapy & 0 & Ref. & 1 & Ref. \\
\hline Penicillin G + gentamicin & 0.68 & {$[-0.10,1.46]$} & 1.39 & {$[0.73,2.66]$} \\
\hline Other antibiotics & 0.33 & {$[-0.43,1.09]$} & 1.92 & {$[1.08,3.42]$} \\
\hline Antibiotic use pre-hospitalization & -0.25 & {$[-0.95,0.46]$} & 1.15 & {$[0.66,1.99]$} \\
\hline Female & 0.08 & {$[-0.53,0.69]$} & 0.50 & {$[0.30,0.82]$} \\
\hline Age & 0.04 & {$[0.02,0.07]$} & 1.01 & {$[0.99,1.03]$} \\
\hline \multicolumn{5}{|l|}{ Comorbidities } \\
\hline COPD & 0.22 & {$[-0.46,0.86]$} & 2.07 & {$[1.26,3.41]$} \\
\hline Heart failure & 1.40 & {$[0.62,2.17]$} & 1.45 & {$[0.83,2.51]$} \\
\hline Diabetes Mellitus I or II & 0.38 & {$[-0.52,1.27]$} & 1.22 & {$[0.63,2.37]$} \\
\hline Nursing home resident & -0.98 & {$[-2.05,2.17]$} & 1.40 & {$[0.64,3.07]$} \\
\hline Year admitted (2012 vs. 2010) & -0.42 & {$[-0.73,-0.11]$} & 0.85 & {$[0.66,1.08]$} \\
\hline \multicolumn{5}{|l|}{ Hospital } \\
\hline Hospital A & 0 & Ref & 1 & Ref. \\
\hline Hospital B & 0.81 & {$[-0,14,1.76]$} & 0.74 & {$[0.37,1.50]$} \\
\hline Hospital C & 1.10 & {$[0.34,1.87]$} & 0.51 & {$[0.29,0.88]$} \\
\hline \multicolumn{5}{|l|}{ Pathogens } \\
\hline None identified & 0 & Ref. & 1 & Ref. \\
\hline S.pneumoniae & 1.22 & {$[0.10,2.34]$} & 0.96 & {$[0.37,2.47]$} \\
\hline H.influenzae & 2.13 & {$[-041,4.67]$} & 3.01 & {$[0.65,14.02]$} \\
\hline M. or C. pneumoniae & 0.75 & {$[-0.98,2.48]$} & 0.39 & {$[0.05,3.22]$} \\
\hline S.aureus & 4.10 & {$[1.24,6.96]$} & 5.24 & {$[0.99,27.6]$} \\
\hline Other bacteria & 5.19 & {$[2.98,7.40]$} & 1.38 & {$[0.26,7.38]$} \\
\hline Influenza virus A or B & 1.86 & {$[-0.83,4.55]$} & - & - \\
\hline Other respiratory viruses & 0.44 & {$[-1.56,2.44]$} & 0.85 & {$[0.10,6.98]$} \\
\hline Two or more pathogens & 1.40 & {$[-1.30,4.10]$} & - & - \\
\hline \multicolumn{5}{|l|}{ CRB-65 score } \\
\hline 0 & 0 & Ref. & 1 & Ref. \\
\hline 1 & -0.19 & {$[-1.24,0.85]$} & 0.82 & {$[0.32,2.06]$} \\
\hline 2 & 0.50 & {$[-0.65,1.64]$} & 0.99 & {$[0.37,2.61]$} \\
\hline 3 & 1.92 & {$[0.35,3.50]$} & 0.71 & {$[0.19,2.66]$} \\
\hline 4 & 1.63 & {$[-2.85,6.11]$} & - & - \\
\hline Missing data & -0.27 & {$[-1.55,1.01]$} & 0.78 & {$[0.26,2.39]$} \\
\hline
\end{tabular}

Cl confidence interval, COPD Chronic obstructive pulmonary disease, CRB-65 Confusion, Respiration, Blood pressure and Age

a Linear regression to explore impact of empirical antibiotic prescribing on length of hospital stay. Adjusted for antibiotic use pre-hospitalization, gender, age, comorbidities, nursing home resident, year admitted (2012 vs. 2010), hospital, pathogens and severity of infection (CRB-65)

${ }^{\mathrm{b}}$ Logistic regression to explore impact of empirical antibiotic prescribing on 30-day readmission. Adjusted for antibiotic use pre-hospitalization, gender, age, comorbidities, nursing home resident, year admitted (2012 vs. 2010), hospital, pathogens and severity of infection (CRB-65)

with COPD had higher risk of readmission [OR 2.07, 95\% CI; 1.26-3.41]. In addition, we found variations in risk of 30-day readmission between the three hospitals. See Table 3 for the complete table with adjusted odds ratio.

\section{Discussion}

In this setting with low levels of AMR among common airway pathogens and extensive use of penicillin $\mathrm{G} / \mathrm{V}$, we found that empirical treatment with penicillin $\mathrm{G} / \mathrm{V}$ in monotherapy was associated with reduced risk of 30-day 
readmission compared to other empirical antibiotic treatments. The 30 -day mortality rate was $6.9 \%$, and the median IV and total treatment duration was 3.0 (mean 3.7 ) and 11.0 days (mean 11.6), respectively.

The extensive use of penicillin G/V in Scandinavian countries is in contrast to other countries where level of penicillin resistance limits the use of penicillin G/V [20]. Whether this approach is associated with favourable clinical outcomes has been sparsely documented until recently. A Danish study found no association between penicillin G/V in monotherapy for non-severe CAP in respect of mortality [3]. As far as we know, association between empirical prescribing with penicillin G/V and risk of readmission has for similar CAP-cohorts not been investigated. Altogether, our findings suggest that prescribing penicillin G/V for non-severe CAP is sound and safe with regard to desired patient outcome (LOS, mortality and readmission). With increasing concerns about AMR and focus on the importance of appropriate antibiotic prescribing, the treatment traditions in Scandinavia and the Netherlands illustrates that it is possible to use narrow spectrum treatments in a setting with low level of AMR.

For severe CAP, the Norwegian CPG recommends penicillin $G$ in combination with gentamicin or cefotaxime in monotherapy. The latter treatment option is also seen in Dutch guidelines for patients with CURB-65 3-5 in non-ICU-settings (i.e. second or third generation cephalosporines) [14]. The evidence for recommending gentamicin is scarce, but gentamicin in combination with penicillin $G$ has been used for severe CAP for decades in Norway [23]. Using gentamicin prevents excessive use of cephalosporines $[14,23]$. The combination therapy covers the main expected pathogens in severe-CAP; gentamicin is primarily efficient in case of bacteraemia covering potential gram-negative pathogens [10]. Penicillin G covers S.pnuemoniae and H.influenzae (in high doses and in absence of resistance) [10].

An aetiological agent (including both viral and bacterial pathogens) was identified in $21 \%$ of patients in our study. This is in agreement with Danish findings by Egelund et al. [3], but low compared to two recent Norwegian studies by Holter et al. [9] and Roysted et al. [8]. These authors found an aetiological agent in 63 and 37\% of CAPpatients. S.pneumoniae was the most prevalent bacterial pathogen both in our study and in the studies by Egelund et al., Holter et al. and Roysted et al. with 9, 5, 30 and $20 \%$, H.influenzae was identified in 2, 4, 5 and $6 \%$ and M.pneumoniae or C.pneumoniae in 3, 3, 6 and 3\%, respectively $[3,8,9]$. While Legionella species was not identified in our study, the Danish study by Egelund et al. identified Legionella species in $<1 \%$. The two Norwegian studies by Holter et al. and Roysted et al. identified
Legionella species in 3 and 6\%, respectively $[8,9]$. Holter et al. describes that nearly all Legionella-cases was infected abroad and Roysted et al. describes that eight of the 21 patients diagnosed with Legionella species was identified by serology post-discharge and these patients recovered without specific Legionella treatment. In addition, some of the patients included in the study by Roysted et al. may be part of a local outbreak in 2008 [24]. Overall, only 40-70 cases have been diagnosed annually with Legionella species in Norway the last 5 years and more than half of the patients are infected abroad [10].

The Norwegian CPG do not recommend empiric antibiotic treatment for atypical pathogens if atypical pathogens are not clinically suspected. This specific recommendation lean on the low incidence of these pathogens and literature that do not show benefit of survival or clinical efficacy for atypical coverage [15]. From our data we have no indication that not covering empirically for atypical pathogens has negative implications in the overall non-severe CAP population.

Studies investigating associations between empirical antibiotic prescribing and clinical outcomes have primarily focused on mortality as outcome measure [25]. Unfortunately, we had too few patients for a conclusive assessment of association with mortality. Still, our data suggest no negative effect on mortality in this patient selection as 30-day mortality in our study (6.9\%) are lower or comparable to other findings from Scandinavia $[3,6]$.

It seems incomprehensible that prescribing antibiotics with broader spectrum should result in more readmission compared to prescribing penicillin G/V in monotherapy. However, after adjusting for relevant covariates, we are still unable to pinpoint the exact reasons for these findings. Whether it has to do with our outcome measure "all-cause readmissions" and not "pneumoniaspecific readmissions" is uncertain, and can unfortunately not be explored as this data has not been collected. However, the advantage of reporting all-cause readmission is that bias inherent with defining exact cause of readmission is avoided [26]. Consequently, applying "all-cause readmission" depends solely on the number of readmissions identified and might therefore be more reliable [26]. Furthermore, the 30-day readmission rate in our study of $14.4 \%$ is comparable to findings in other studies $[4,5]$.

In an observational study it is challenging to attribute causality to an observed association. The selection of statistical model is critical for minimizing bias in estimates when testing association between exposure and outcome. A strength in our study is that we have applied DAGs to structurally approach the minimal set of covariates to include in the model, and we thereby increase statistical efficiency. In addition, applying DAGs to guide 
assumptions for the regression models increase transparency. Possibly, by collecting more information on comorbidities, we could further reduce bias in our models. CRB-65, an indicator of severity, does not significantly differ between the groups prescribed penicillin G/V and other antibiotics. Still, due to the retrospective design we cannot rule out selection bias and confounding by indication. Consequently, we cannot rule out that patients treated with penicillin G/V had less severe CAP compared to patients prescribed other antibiotics.

We have identified several areas with room for improvement. One of them is the high level of recorded penicillin allergy $(10.8 \%)$ which is not in accordance with the estimated prevalence of $<1 \%$ [27]. Penicillin allergy testing should be standard care in hospitals, and is increasingly integrated in antibiotic stewardship programs globally [28]. Hospitals that have implemented delabelling activities have had success in reducing prescription of restricted antibiotics, and it is proven to be safe for the patients $[29,30]$.

Median IV treatment duration was 3.0 days, which may be considered adequate. However, we suspect that IV treatment duration could have been shorter because our study population mainly comprised non-severe CAP-patients.

A total of 10-14 days antibiotic treatment duration seems to have gained wide acceptance [31,32], and this is in line with our findings. This is significantly longer than the CPG-recommended duration of 5-7 and 7-10 days in non-severe and severe CAP-patients, respectively. In addition, recent literature indicates that duration as short as 3 days is non-inferior to longer treatment [33].

Our study has several methodological strengths and limitations. First, we have a homogenous study population and consequently a relative precise estimate of the clinical outcomes in the selected population. Second, this is a retrospective observational study and there will always be a risk of bias due to unmeasured variables. Third, the scarcity in patient records of data on infection relevant clinical- and laboratory data from the first 3 days of the hospital stay refrained assessment of time to clinical stability. Fourth, CRB-65 is recommended as a scoring tool for severity in Norway [15]. Surprisingly, we observed that CRB-65 score was documented in only one patient record. Consequently, we had to calculate CRB-scores based on information in admission notes. Our classification of severity of disease may be in conflict with physicians' judgment at time of empirical prescribing. Still, the distribution of CRB-65 scores is comparable to other studies, with a substantial proportion of patients with low risk of mortality [34]. Fifth, if we had collected data on ICU-admissions, our assumptions on severity could have been strengthened. On the other hand, using ICU-admission as a surrogate for severity is not unproblematic. The decision to admit a patient to an ICU can be due to other considerations than severity and can vary widely between hospitals [35].

\section{Conclusion}

In a Norwegian hospital setting predominated by nonsevere CAP patients, we found that prescribing penicillin G/V was associated with lower risk of 30-day readmission compared to other antibiotic treatments. Our results support the national guideline recommendations for empirical antibiotic prescribing for patients presenting without complicating factors such as immunosuppression and frequent readmissions. Our data can be used to reassure clinicians that this treatment is appropriate in this specific setting and patient population.

The proportion of patients with penicillin allergy was high and median total treatment duration unnecessary long.

\section{Supplementary information}

Supplementary information accompanies this paper at https://doi.org/10. 1186/s12890-020-01188-6.

Additional file 1. Directed Acyclic Graphs; Association between empirical prescribing (exposure) and readmission or length of stay in hospital (outcomes).

Additional file 2. Description of all empirical antibiotics prescribed

Additional file 3. Description of empirical antibiotic prescribed versus subsequent bacterial pathogen identified

\section{Abbreviations}

AMR: Antimicrobial resistance; CAP: Community-acquired pneumonia; CPG: Clinical practice guideline; COPD: Chronic obstructive pulmonary disease; CRB-65: Confusion, respiratory rate, blood pressure, age $\geq 65$ years; DAGs: Directed Acyclic Graphs; IV: Intravenous; LOS: Length of stay in hospital; UNN: University Hospital of NorthNorway

\section{Acknowledgements}

The publication charges for this article have been funded by a grant from the publication fund of UiT The Arctic University of Norway.

\section{Authors' contributions}

$\mathrm{JUH}$; planning and performing the study, analysis of data, writing of manuscript, BHG; planning the study, writing of manuscript, KS; statistical analysis, interpretation of statistical results, writing of manuscript, VS; planning the study, writing of manuscript, LS; planning the study, writing of manuscript. The author(s) read and approved the final manuscript.

\section{Funding}

This research received no specific grant from any funding agency. The study was conducted as a part of a PhD-education, funded by UiT The Arctic University of Norway.

\section{Availability of data and materials}

The datasets generated and/or analysed during the current study are not publicly available, but are available from the corresponding author on reasonable request.

\section{Ethics approval and consent to participate}

The Regional Committee for Medical and Health Research Ethics approved the final protocol and concluded that written consent from patients was not required. All data are reported aggregated and anonymously, and patient confidentially is maintained. The Data Protection Officer at UNN had no objection to the final protocol, and approved the study. 


\section{Consent for publication}

Not applicable.

\section{Competing interests}

The authors declare that they have no competing interests.

\section{Author details}

${ }^{1}$ Regional Centre for Infection Control, University Hospital of North Norway, N-9038 Troms $\varnothing$, Norway. ${ }^{2}$ Department of Pharmacy, Faculty of Health Sciences, UiT - The Arctic University of Norway, N-9037 Tromsø, Norway. ${ }^{3}$ Hospital Pharmacy of North Norway Trust, N-9291 Tromsø, Norway. ${ }^{4}$ Department of Infectious Diseases, Division of Internal Medicine, University Hospital of North Norway, N-9038 Tromsø, Norway. ${ }^{5}$ Department of Clinical Medicine, Faculty of Health Sciences, UiT - The Arctic University of Norway, N-9037 Tromsø, Norway. ${ }^{6}$ Infectious Diseases Unit, LaFe University Hospital, Valencia, Spain.

Received: 13 September 2019 Accepted: 15 May 2020

Published online: 15 June 2020

\section{References}

1. Torres A, Peetermans WE, Viegi G, Blasi F. Risk factors for communityacquired pneumonia in adults in Europe: a literature review. Thorax. 2013; 68(11):1057-65.

2. Torres A, Cilloniz C, Blasi F, Chalmers JD, Gaillat J, Dartois N, et al. Burden of pneumococcal community-acquired pneumonia in adults across Europe: a literature review. Respir Med. 2018;137:6-13.

3. Egelund $G B$, Jensen $A V$, Andersen $S B$, Petersen PT, Lindhardt $B O$, von Plessen $C$, et al. Penicillin treatment for patients with community-acquired pneumonia in Denmark: a retrospective cohort study. BMC Pulm Med. 2017; 17(1):66.

4. Mather JF, Fortunato GJ, Ash JL, Davis MJ, Kumar A. Prediction of pneumonia 30-day readmissions: a single-center attempt to increase model performance. Respir Care. 2014;59(2):199-208.

5. Hadfield J, Bennett L. Determining best outcomes from communityacquired pneumonia and how to achieve them. Respirology. 2018;23(2): 138-47

6. Dwyer R, Hedlund J, Henriques-Normark B, Kalin M. Improvement of CRB-65 as a prognostic tool in adult patients with community-acquired pneumonia. BMJ Open Respir Res. 2014;1(1):e000038.

7. Carbonara S, Stano F, Scotto G, Monno L, Angarano G. The correct approach to community-acquired pneumonia in immunocompetent adults: review of current guidelines. New Microbiol. 2008;31(1):1-18.

8. Roysted W, Simonsen O, Jenkins A, Sarjomaa M, Svendsen MV, Ragnhildstveit $E$, et al. Etiology and risk factors of community-acquired pneumonia in hospitalized patients in Norway. Clin Respir J. 2016;10(6):756-64.

9. Holter JC, Muller F, Bjorang O, Samdal HH, Marthinsen JB, Jenum PA, et al. Etiology of community-acquired pneumonia and diagnostic yields of microbiological methods: a 3-year prospective study in Norway. BMC Infect Dis. 2015;15(1):64.

10. NORM/NORM-VET 2017 and 2018. Usage of antimicrobial agents and occurrence of antimicrobial resistance in Norway. Tromsø/Oslo: ISSN:18909965 (electronic)

11. Eccles S, Pincus C, Higgins B, Woodhead M, Guideline Development Group. Diagnosis and management of community and hospital acquired pneumonia in adults: summary of NICE guidance. BMJ. 2014;349:g6722.

12. Woodhead M, Blasi F, Ewig S, Garau J, Huchon G, leven M, et al. Guidelines for the management of adult lower respiratory tract infections--full version. Clin Microbiol Infect. 2011;17(Suppl 6):E1-59.

13. Metlay JP, Waterer GW, Long AC, Anzueto A, Brozek J, Crothers K, et al. Diagnosis and treatment of adults with community-acquired pneumonia. An Official Clinical Practice Guideline of the American Thoracic Society and Infectious Diseases Society of America. Am J Respir Crit Care Med. 2019; $1(200(7)):$ e45-67.

14. Wiersinga WJ, Bonten MJ, Boersma WG, Jonkers RE, Aleva RM, Kullberg BJ, et al. Management of community-acquired pneumonia in adults: 2016 guideline update from the Dutch working party on antibiotic policy (SWAB) and Dutch Association of Chest Physicians (NVALT). Neth J Med. 2018;76(1):4-13.

15. The Norwegian Directorate of Health. National clinical guideline for use of antibiotics in hospital (in Norwegian). 2013. Available from http://www. helsedirektoratet.no/retningslinjer/antibiotika-i-sykehus Accessed 13 Feb 2020.

16. Johansen IS, Gerstoft J, Engberg JH, Frimodt-Møller N, Böcher S, Nygaard U, et al. Pneumoni. Available from http://www.pro.medicin.dk/Specielleemner/ Emner/3185772018. Accessed 13 Feb 2020.

17. Athlin S, Hedlund J, Lidman C, Lundqvist A, Naucler P, Nilsson A, et al. Vårdprogram för samhällsförvärvad pneumoni. Available from http://www. infektion.net/wp-content/uploads/2017/05/vardprogram_pneumoni_2016. pdf. Accessed 13 Feb 2020.

18. World Health Organization. Antimicrobial resistance: global report on surveillance, 2014

19. Goossens H. Antibiotic consumption and link to resistance. Clin Microbiol Infect. 2009;15(Suppl 3):12-5.

20. Athanassa Z, Makris G, Dimopoulos G, Falagas ME. Early switch to oral treatment in patients with moderate to severe community-acquired pneumonia: a meta-analysis. Drugs. 2008;68(17):2469-81.

21. Bauer T, Ewig S, Marre R, Suttorp N, Welte T. CRB-65 predicts death from community-acquired pneumonia. J Intern Med. 2006;260(1):93-101.

22. Shrier I, Platt RW. Reducing bias through directed acyclic graphs. BMC Med Res Methodol. 2008:8:70.

23. Lindemann PC, Haldorsen BC, Smith I, Sjursen H, Mylvaganam $H$. Aminoglycosides should still be used in empirical sepsis treatment. Tidsskr Nor Laegeforen. 2013;133(10):1054

24. Norwegian Institute of Public Health. Outbreak of Legionella in Norway. Available from: www.fhi.no/sv/utbrudd/oversikt-over-storre-utbrudd/ utbrudd-av-legionellose-i-norge/. Accessed 13 Feb 2020.

25. Schuts EC, Hulscher M, Mouton JW, Verduin CM, Stuart J, Overdiek H, et al. Current evidence on hospital antimicrobial stewardship objectives: a systematic review and meta-analysis. Lancet Infect Dis. 2016;16(7):847-56.

26. Penston J, Steele RJC, Brewster D. Should we use total mortality rather than cancer specific mortality to judge cancer screening programmes? BMJ. 2011;343:d6397.

27. Solensky R. Hypersensitivity reactions to beta-lactam antibiotics. Clin Rev Allergy Immunol. 2003;24(3):201-20.

28. Sacco KA, Bates A, Brigham TJ, Imam JS, Burton MC. Clinical outcomes following inpatient penicillin allergy testing: a systematic review and metaanalysis. Allergy. 2017:72(9):1288-96.

29. Devchand M, Kirkpatrick CMJ, Stevenson W, Garrett K, Perera D, Khumra S, et al. Evaluation of a pharmacist-led penicillin allergy de-labelling ward round: a novel antimicrobial stewardship intervention. J Antimicrob Chemother. 2019;74(6):1725-30.

30. du Plessis T, Walls G, Jordan A, Holland DJ. Implementation of a pharmacistled penicillin allergy de-labelling service in a public hospital. J Antimicrob Chemother. 2019:74(5):1438-46.

31. Vaughn VM, Flanders SA, Snyder A, Conlon A. Excess antibiotic treatment duration and adverse events in patients hospitalized with pneumonia: a multihospital cohort study. Ann Intern Med. 2019;171(3):153-63.

32. Aliberti S, Blasi F, Zanaboni AM, Peyrani P, Tarsia P, Gaito S, et al. Duration of antibiotic therapy in hospitalised patients with community-acquired pneumonia. Eur Respir J. 2010;36(1):128-34.

33. el Moussaoui $R$, de Borgie CA, van den Broek P, Hustinx WN, Bresser P, van den Berk GE, et al. Effectiveness of discontinuing antibiotic treatment after three days versus eight days in mild to moderate-severe community acquired pneumonia: randomised, double blind study. BMJ. 2006; 10(332(7554)):1355.

34. Swedish Society of Infectious Diseases, ed. Naucler P. Quality registre for pneumonia, annual report 2013. Available from http://www.infektion.net/ sites/default/files/pneumoni_2013.pdf. Accessed 13 Feb 2020.

35. Wunsch H, Angus DC, Harrison DA, Walter T, Linde-Zwirble WT, Rowan KM Comparison of medical admissions to intensive care units in the United States and United Kingdom. Am J Respir Crit Care Med. 2011;183(12):1666-73.

\section{Publisher's Note}

Springer Nature remains neutral with regard to jurisdictional claims in published maps and institutional affiliations. 